\title{
Contraction par Frobenius et modules de Steinberg
}

\author{
Michel Gros et Kaneda Masaharu
}

\begin{abstract}
Résumé. Soit $G$ un groupe réductif défini sur un corps algébriquement clos de caractéristique positive. Nous montrons que le foncteur contraction par Frobenius de la catégorie des $G$-modules est adjoint à droite de celui de tensorisation deux fois par le module de Steinberg du tordu de Frobenius du $G$-module de départ. Il s'ensuit en particulier que le foncteur de contraction par Frobenius préserve le caractère injectif et l'existence de bonne filtration mais pas la semi-simplicité.

For a reductive group $G$ defined over an algebraically closed field of positive characteristic, we show that the Frobenius contraction functor of $G$-modules is right adjoint to the Frobenius twist of the modules tensored with the Steinberg module twice. It follows that the Frobenius contraction functor preserves injectivity, good filtrations, but not semisiplicity.
\end{abstract}

Soit $G$ un groupe algébrique réductif défini sur un corps algébriquement clos $\mathbb{k}$ de caractéristique $p>0$. Le morphisme induit sur l'algèbre des distributions $\operatorname{Dist}(G)$ de $G$ par l'endomorphisme de Frobenius de $G$ admet un scindage [GK11], [GK15]. Ce dernier permet de définir une nouvelle opération sur les $G$-modules, qu'à la suite de Littelmann qui l'avait défini auparavant seulement pour les modules de Weyl duaux, nous appellons contraction par Frobenius. Si $M$ est un $G$-module, la contraction par Frobenius permet de munir la somme directe des sous-espaces de $M$ de poids divisible par $p$ d'une structure de $G$-module. Cette construction généralise le processus de "détorsion" par Frobenius sur $M$ noté $M^{[-1]}$ dans [J, II.3.16], lorsque l'on part d'une situation où le noyau de Frobenius de $G$ agit trivialement sur $M$.

Nous présentons tout d'abord dans cet article la caractérisation, dégagée par Stephen Donkin, du foncteur contraction par Frobenius comme adjoint à droite de celui de double tensorisation par le module de Steinberg du tordu par Frobenius (2.3, Cor.). Ceci nous permet ensuite d'établir inconditionnellement quelques pro-

Le second auteur a bénéficié lors de ce travail d'un soutien JSPS Grants in Aid for Scientific Research 15K04789. 
priétés du foncteur contraction par Frobenius: préservation du caractère injectif et de l'existence d'une bonne filtration pour les $G$-modules $(3.1$, Th.) mais pas de la semi-simplicité en général (1.4). Enfin, nous démontrons des résultats similaires $\left(4^{\circ}\right)$ pour les $G_{r}$-modules, $G_{r}$ désignant le $r$-ième noyau de Frobenius de $G$.

Cet article tire son origine d'une question posée à S. Donkin par le second auteur (M.K.) lors de sa visite à l'Institut Mittag-Leffler en Mai 2015. Il remercie cet Institut de lui avoir procuré cette opportunité ainsi que Donna Testerman de l'avoir invité à exposer une partie de ce travail à l'Institut Bernouilli en Août 2016. Les auteurs remercient chaleureusement S. Donkin de leur avoir permis d'inclure l'un de ses énoncés et assument la pleine responsabilité d'éventuelles erreurs dans la démonstration que nous en présentons ici. Les auteurs sont également très reconnaissants à H. H. Andersen de leur avoir signalé une bévue dans une formulation antérieure de la proposition 1.3 ainsi qu'aux referees pour leurs très bénéfiques suggestions.

\section{$1^{\circ}$. Scindage du Frobenius de $\operatorname{Dist}(G)$}

Soit $G$ comme ci-dessus supposé également simplement connexe et semi-simple. Par commodité, nous supposerons de plus fixée une $\mathbb{F}_{p}$-forme $G_{\mathbb{F}_{p}}$ de $G$ et noterons simplement $\mathrm{F}$ le morphisme de Frobenius (géométrique) correspondant de $G$ défini par ? ${ }^{p} \otimes_{\mathbb{F}_{p}} \mathbb{k}$ sur son algèbre des coordonnées $\mathbb{k}[G]=\mathbb{F}_{p}\left[G_{\mathbb{F}_{p}}\right] \otimes_{\mathbb{F}_{p}} \mathbb{k}[\mathrm{J}$, I.9.2]. Soient $B$ un sous-groupe de Borel de $G, T$ un tore maximal de $B$ tous deux scindés sur $\mathbb{F}_{p}, R$ le système de racines de $G$ relativement à $T, R^{+}$la partie positive de $R$ pour laquelle les racines de $B$ sont $-R^{+}$, et $R^{\text {s }}$ l'ensemble des racines simples de $R^{+}$. Pour tout sous-schéma en groupes $H$ de $G, \operatorname{Dist}(H)$ désignera l'algèbre des distributions sur $H$, et $H_{1}$ le noyau de Frobenius de $H$.

1.1. Comme $T$ est abélien, l'unité de $\operatorname{Dist}\left(T_{1}\right)$ admet une unique décomposition en idempotents primitifs orthogonaux [NT, §1. Thm. 4.6]. Parmi ces derniers, il en existe un unique $\mu_{0}$ à ne pas être annulé par la co-unité de $\operatorname{Dist}\left(T_{1}\right)$ et c'est aussi l'unique idempotent à ne pas être annulé par $\operatorname{Dist}(\mathrm{F}) ; \operatorname{Dist}(\mathrm{F})\left(\mu_{0}\right)=1$. Soient $x_{\alpha}$, $\alpha \in R, h_{\beta}, \beta \in R^{\mathrm{s}}$, une base de Chevalley de l'algèbre de Lie de $G$ correspondant à $T$ et aux sous-groupes radiciels de $G$. Pour $n \in \mathbb{N}$, nous noterons $x_{\alpha}^{(n)}$ la $n$-ième puissance divisée de $x_{\alpha}$. Il existe un homomorphisme de $\mathbb{k}$-algèbres $\phi: \operatorname{Dist}(G) \rightarrow \mu_{0} \operatorname{Dist}(G) \mu_{0}$ tel que $x_{ \pm \alpha}^{(n)} \mapsto \mu_{0} x_{ \pm \alpha}^{(p n)} \mu_{0},\left(\begin{array}{c}h_{\alpha} \\ m\end{array}\right) \mapsto \mu_{0}\left(\begin{array}{c}h_{\alpha} \\ p m\end{array}\right) \mu_{0}$ pour tous $\alpha \in R^{\mathrm{s}}, n \in \mathbb{N}$ (cf. [J, I.7.8] pour les notations), et vérifiant $\operatorname{Dist}(\mathrm{F}) \circ \phi=\operatorname{id}_{\operatorname{Dist}(G)}[\mathrm{GK} 11]$, [GK15]. Nous appellerons $\phi$ le scindage du Frobenius sur $\operatorname{Dist}(G)$.

Etant donné un $G$-module $M$, en faisant agir $\operatorname{Dist}(G)$ sur $M$ via $\phi$, i.e. sur $\mu_{0} M$, on obtient ainsi un nouveau $\operatorname{Dist}(G)$-module. Comme $M$ est localement fini, il en est de même de $\mu_{0} M$, et l'on obtient donc ([J, II.1.20]) une structure de 
$G$-module sur $\mu_{0} M$ [J, II.1.20], qu'on appellera la contraction par Frobenius de $M$ et qu'on notera $M^{\phi}$. L'action de $\operatorname{Dist}(G)$ sur $M^{\phi}$ sera notée par $\bullet_{\phi}: \mu_{\phi} m=\phi(\mu) m$, $\mu \in \operatorname{Dist}(G), m \in M^{\phi}$.

1.2. Rappelons maintenant quelques propriétés basiques de cette contraction par Frobenius. Comme $\mu_{0}$ est un idempotent,

(i) le foncteur de contraction par Frobenius est exact.

Soit $\Lambda$ le groupe des caractères de $T$. Pour tout $\lambda \in \Lambda$, nous noterons encore abusivement $\lambda$ l'application $\operatorname{Dist}(\lambda): \operatorname{Dist}(T) \rightarrow \mathbb{k}$. Soit $M$ un $G$-module. Si $M_{\lambda}=\{m \in$ $M \mid t m=\lambda(t) m, \forall t \in T\}=\{m \in M \mid \mu m=\lambda(\mu) m, \forall \mu \in \operatorname{Dist}(T)\}$ désigne le sous-espace de poids $\lambda$ de $M$, on a:

(ii) $\mu_{0} M=\coprod_{\lambda \in \Lambda} M_{p \lambda}$ avec $M_{p \lambda}$ l'espace de poids $\lambda$ de $M^{\phi}$; considérant $m \in M_{p \lambda}$ comme un élément de $M^{\phi}$, on a $\left(\begin{array}{c}h_{\beta} \\ n\end{array}\right) \bullet_{\phi} m=\left(\left\langle\lambda, \beta^{\vee}\right\rangle\right) m$ pour tous $\beta \in R^{\mathrm{s}}$ et $n \in \mathbb{N}$. En particulier, si $\sum_{\lambda \in \Lambda} M_{p \lambda}=0$, la contraction par Frobenius annule $M$.

(iii) Si l'on fait agir $G$ sur $M$ via le morphisme de Frobenius de $G$, on obtient un nouveau $G$-module appelé le tordu par Frobenius de $M$ et noté $M^{[1]}$. La contraction par Frobenius de ce dernier redonne alors $M:\left(M^{[1]}\right)^{\phi} \simeq M$. En particulier, le foncteur de torsion par Frobenius $M \mapsto M^{[1]}$ est un endofoncteur pleinement fidèle de la catégorie des $G$-modules.

(iv) Si $G_{1}$ agit trivialement sur $M$, la $G$-action sur $M$ se factorise par le quotient $G \rightarrow G / G_{1}$, que l'on peut identifier au morphisme de Frobenius grâce au diagramme commutatif:

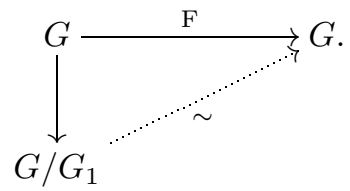

Il s'ensuit qu'il existe une structure de $G$-module, notée $M^{[-1]}$ dans [J, II.3.16], sur le $\mathbb{k}$-espace vectoriel $M$ détordant la torsion par Frobenius, et que l'on retrouve le $G$-module $M$ de départ en tordant par Frobenius: $\left(M^{[-1]}\right)^{[1]} \simeq M$. Le $G$-module $M^{[-1]}$ n'est donc alors rien d'autre dans ce cas que la contraction par Frobenius $M^{\phi}$ de $M$.

(v) La contraction par Frobenius commute avec la formation du dual: $\left(M^{\phi}\right)^{*} \simeq$ $\left(M^{*}\right)^{\phi}$ pour tout $M$ de dimension finie [GK15, 5.5], [GK, Prop. 6.6].

(vi) Si $V$ est un $G$-module, $\operatorname{Dist}(G)$ agit sur $M \otimes V$ via la comultiplication $\Delta$ de $\operatorname{Dist}(G)$; si $\Delta(x)=\sum_{i} x_{i} \otimes y_{i}$ et si $x \in \operatorname{Dist}(G)$, alors $x .(m \otimes v)=\sum_{i} x_{i} m \otimes y_{i} v, m \in M$, $v \in V$. Bien que la contraction par Frobenius ne commute pas avec la comultiplication, on a [GK, Lem. 6.8]:

$$
\left(M \otimes V^{[1]}\right)^{\phi} \simeq M^{\phi} \otimes V, \quad\left(M^{[1]} \otimes V\right)^{\phi} \simeq M \otimes V^{\phi} .
$$


1.3. Soit $\Lambda^{+}=\left\{\lambda \in \Lambda \mid\left\langle\lambda, \alpha^{\vee}\right\rangle \geq 0, \forall \alpha \in R^{+}\right\}$l'ensemble des poids dominants. Les $G$-modules simples sont paramétrisés par leur plus haut poids dominant; notons alors $L(\lambda)$ le $G$-module simple de plus haut poids $\lambda$. Si $\Lambda_{1}=\left\{\mu \in \Lambda^{+} \mid\left\langle\mu, \alpha^{\vee}\right\rangle<p, \forall \alpha \in\right.$ $R^{\mathrm{s}}$, le théorème de Steinberg de décomposition en produit tensoriel [J, II.3.17] donne un isomorphisme de $G$-modules $L(\lambda) \simeq L\left(\lambda^{0}\right) \otimes L\left(\lambda^{1}\right)^{[1]} \otimes \ldots \otimes L\left(\lambda^{r}\right)^{[r]}, \lambda^{i} \in \Lambda_{1}$ avec $\lambda=\sum_{i=0}^{r} p^{i} \lambda^{i}$, et $?^{[j]}$ la torsion par $F^{j}$. On a donc

$$
L(\lambda)^{\phi} \simeq L\left(\lambda^{0}\right)^{\phi} \otimes L\left(\lambda^{1}\right) \otimes L\left(\lambda^{2}\right)^{[1]} \otimes \ldots \otimes L\left(\lambda^{r}\right)^{[r-1]} .
$$

Une estimation grossière des poids donne alors

Proposition. Soit h le maximum des nombres de Coxeter des composantes simples de $G$. Supposons que $p \geq 2 h-3$. Un G-module simple de plus haut poids appartenant à $\Lambda_{1}$ se contracte par Frobenius en un G-module semi-simple si ce dernier est non nul.

Preuve. On peut supposer $G$ simple. Soit $\lambda \in \Lambda_{1}$, et soit $p \mu, \mu \in \Lambda^{+}$, un poids de $L(\lambda)$. On a alors $p \mu=\lambda-\gamma$ pour un certain $\gamma \in \sum_{\alpha \in R^{\mathrm{s}}} \mathbb{N} \alpha$. Si $\alpha_{0}^{\vee}$ est la plus grande coracine de $R^{\vee}$,

$$
\begin{aligned}
\left\langle\mu+\rho, \alpha_{0}^{\vee}\right\rangle & =\left\langle\frac{1}{p}(\lambda-\gamma)+\rho, \alpha_{0}^{\vee}\right\rangle=\frac{1}{p}\left\langle\lambda-\gamma, \alpha_{0}^{\vee}\right\rangle+(h-1) \\
& \leq \frac{1}{p}\left\langle\lambda, \alpha_{0}^{\vee}\right\rangle+(h-1) \leq \frac{1}{p}\left\langle(p-1) \rho, \alpha_{0}^{\vee}\right\rangle+(h-1) \\
& =\frac{1}{p}(p-1)(h-1)+(h-1)=(h-1)\left(2-\frac{1}{p}\right)<2(h-1) \leq p .
\end{aligned}
$$

Comme $\left\langle\mu+\rho, \alpha_{0}^{\vee}\right\rangle \in \mathbb{N}$, on doit en fait avoir $\left\langle\mu+\rho, \alpha_{0}^{\vee}\right\rangle \leq 2 h-3 \leq p$. L'assertion est alors une conséquence du linkage principle [J, II.6.17].

\subsection{Remarques.}

(i) Le même argument montre que si $M$ est un $G$-module dont tous les poids dominants sont de la forme $p \lambda$ avec $\lambda \in \Lambda_{1}$, alors $M$ est semi-simple.

(ii) La conclusion de la proposition est inexacte sans hypothèse de caractéristique ou de petitesse du plus haut poids comme on le montrera en (3.4). D'autre part, prendre les points fixes sous $G_{1}$ d'un module semi-simple préserve, si cela ne l'annule pas, la semi-simplicité.

\section{$2^{\circ}$. Caractérisation par les modules de Steinberg}

Pour un $G$-module $M$ et pour un sous-schéma en groupes $H$ de $G$ on note $M^{H}$ l'ensemble des éléments $H$-invariants de $M$. Comme $G_{1}$ est normal dans $G$, 
$M^{G_{1}}$ est $G$-sous-module de $M$. Soit $\mathrm{St}=L((p-1) \rho), \rho=\frac{1}{2} \sum_{\alpha \in R^{+}} \alpha$, le module de Steinberg de $G$. Il est auto-dual.

S'il n'y a pas de risque d'ambiguité, nous noterons encore simplement $\nu \in \Lambda$ la $B$-représentation de dimension 1 attachée canoniquement à $\nu$. Pour deux objets $A, B$ d'une catégorie $\mathcal{C}$, nous noterons désormais $\mathcal{C}(A, B)$ l'ensemble des morphismes de $A$ vers $B$. Nous désignerons par $H$ Mod (resp. Dist $(H)$ Mod) la catégorie des $H$-modules rationnels (resp. Dist $(H)$-modules).

2.1. Nous nous proposons maintenant de donner une preuve de l'énoncé suivant.

Théorème. (Donkin) Pour tout G-module $M$, il existe un isomorphisme de G-modules

$$
M^{\phi} \simeq\left\{(\mathrm{St} \otimes \mathrm{St} \otimes M)^{G_{1}}\right\}^{[-1]} .
$$

Preuve. Fixons $v_{+}$(resp. $v_{-}$) un vecteur de plus haut (resp. bas) poids de St. Il résulte alors de [D, Lem. 5.1] que $\operatorname{St} \otimes$ St est engendré $\operatorname{sur} \operatorname{Dist}\left(G_{1}\right)$ par $v_{+} \otimes v_{-}$. L'application $G_{1}$-linéaire $\theta: \operatorname{Dist}\left(G_{1}\right) \otimes_{\operatorname{Dist}\left(T_{1}\right)} \mu_{0} \rightarrow \operatorname{St} \otimes$ St définie par $x \otimes 1 \mapsto x\left(v_{+} \otimes v_{-}\right)$est donc surjective, et par suite bijective pour une raison de dimension. L'application $\theta$ permet de munir $\mu_{0} M$ d'une structure de $G$-module, via les isomorphismes $\mathbb{k}$-linéaires

$$
\begin{aligned}
\mu_{0} M & \simeq \operatorname{Dist}\left(T_{1}\right)\left(\mu_{0}, M\right) \simeq \operatorname{Dist}\left(G_{1}\right) \operatorname{Mod}\left(\operatorname{Dist}\left(G_{1}\right) \otimes_{\operatorname{Dist}\left(T_{1}\right)} \mu_{0}, M\right) \\
& \simeq G_{1} \operatorname{Mod}(\operatorname{St} \otimes \operatorname{St}, M),
\end{aligned}
$$

tels que

$$
\mu_{0} M \ni m \longmapsto f_{m} \in G_{1} \operatorname{Mod}(\operatorname{St} \otimes \mathrm{St}, M) \quad \text { avec } \quad f_{m}\left(v_{+} \otimes v_{-}\right)=m .
$$

Pour $x \in \operatorname{Dist}\left(G_{1}\right)$, on a $f_{m}\left(x\left(v_{+} \otimes v_{-}\right)\right)=x m$ avec $x$ agissant sur $m$ dans le membre de droite via la structure de $G$-module sur $M$.

Comme $(\operatorname{St} \otimes \operatorname{St} \otimes M)^{G_{1}} \simeq G_{1} \operatorname{Mod}(\operatorname{St} \otimes \operatorname{St}, M)$ par auto-dualité de St, on va montrer que ce dernier est isomorphe à $\left(M^{\phi}\right)^{[1]}$ comme $G$-module. On est ainsi réduit à montrer que l'application

$$
\eta:\left(M^{\phi}\right)^{[1]} \longrightarrow G_{1} \operatorname{Mod}(\operatorname{St} \otimes \operatorname{St}, M) \quad \text { via } \quad m \longmapsto f_{m}
$$

est $G$-linéaire. Comme $\operatorname{Dist}(G)$ est engendré par les $x_{ \pm \alpha}^{(n)}, \alpha \in R^{\mathrm{s}}, n \in \mathbb{N}[\mathrm{J} 73$, Satz I.7], il suffit de vérifier que

$$
\eta\left(x_{ \pm \alpha}^{(n)} . m\right)=x_{ \pm \alpha}^{(n)} \eta(m)
$$

expression dans laquelle . dans le membre de gauche désigne l'action usuelle de $x_{\alpha}^{(n)} \operatorname{sur}\left(M^{\phi}\right)^{[1]}$. Rappelons que l'action de $\operatorname{Dist}(G) \operatorname{sur} G_{1} \operatorname{Mod}\left(M, M^{\prime}\right)$ pour 
des $G$-modules $M$ et $M^{\prime}$ est telle que pour $f \in G_{1} \operatorname{Mod}\left(M, M^{\prime}\right)$ et $\beta \in R$, on ait $x_{\beta}^{(n)} f(m)=\sum_{i=0}^{n} x_{\beta}^{(n-i)} f\left((-1)^{i} x_{\beta}^{(i)} m\right)$ pour tout $m \in M[\mathrm{~J}$, I.7.8, 11] grâce à la bijection $\left(M^{*} \otimes M^{\prime}\right)^{G_{1}} \rightarrow G_{1} \operatorname{Mod}\left(M, M^{\prime}\right)$ via $\gamma \otimes m^{\prime} \mapsto \gamma(?) m^{\prime}$.

On a donc

$$
\begin{aligned}
& \left(x_{\alpha}^{(n)} f_{m}\right)\left(v_{+} \otimes v_{-}\right) \\
& \quad=\sum_{b \in[0, n]} x_{\alpha}^{(n-b)} f_{m}\left((-1)^{b} x_{\alpha}^{(b)}\left(v_{+} \otimes v_{-}\right)\right) \\
& =\sum_{b \in[0, n]} x_{\alpha}^{(n-b)} f_{m}\left((-1)^{b} \sum_{s \in[0, b]}\left(x_{\alpha}^{(s)} \otimes x_{\alpha}^{(b-s)}\right)\left(v_{+} \otimes v_{-}\right)\right) \\
& =\sum_{b \in[0, n]}(-1)^{b} x_{\alpha}^{(n-b)} f_{m}\left(v_{+} \otimes x_{\alpha}^{(b)} v_{-}\right) \\
& \text {car } v_{+} \text {est un vecteur de plus haut poids de St }
\end{aligned}
$$$$
=\sum_{b \in[0, p[}(-1)^{b} x_{\alpha}^{(n-b)} f_{m}\left(v_{+} \otimes x_{\alpha}^{(b)} v_{-}\right)
$$$$
\text { car }-(p-1) \rho+b \alpha \text { n'est pas un poids de St pour } b \geq p
$$$$
=\sum_{b \in[0, p[}(-1)^{b} x_{\alpha}^{(n-b)} f_{m}\left(x_{\alpha}^{(b)}\left(v_{+} \otimes v_{-}\right)\right)
$$

car, à nouveau, $v_{+}$est un vecteur de plus haut poids de St

$=\sum_{b \in[0, p[}(-1)^{b} x_{\alpha}^{(n-b)} x_{\alpha}^{(b)} f_{m}\left(v_{+} \otimes v_{-}\right) \quad$ car $f_{m}$ est $G_{1}$-linéaire

$$
=\sum_{b \in[0, p[}(-1)^{b}\left(\begin{array}{l}
n \\
b
\end{array}\right) x_{\alpha}^{(n)} m
$$

$=\sum_{b \in[0, p[}(-1)^{b}\left(\begin{array}{c}n_{0} \\ b\end{array}\right) x_{\alpha}^{(n)} m$ en écrivant $n=n_{0}+p n_{1}$ avec $n_{0} \in\left[0, p\left[\right.\right.$ et $n_{1} \in \mathbb{N}$

$=\sum_{b \in\left[0, n_{0}\right]}(-1)^{b}\left(\begin{array}{c}n_{0} \\ b\end{array}\right) x_{\alpha}^{(n)} m=(1-1)^{n_{0}} x_{\alpha}^{(n)} m= \begin{cases}x_{\alpha}^{(n)} m & \text { si } p \mid n \\ 0 & \text { sinon. }\end{cases}$

De même avec $x_{-\alpha}^{(n)}$, et donc

$$
x_{ \pm \alpha}^{(n)} f_{m}= \begin{cases}f_{x_{ \pm \alpha}^{(n)} m} & \text { si } p \mid n \\ 0 & \text { sinon. }\end{cases}
$$

D'autre part, pour $m \in\left(M^{\phi}\right)^{[1]}$, on a

$$
x_{ \pm \alpha}^{(n)} \bullet m=\operatorname{Dist}(\mathrm{F})\left(x_{ \pm \alpha}^{(n)}\right) \bullet{ }_{\phi} m
$$




$$
\begin{aligned}
& = \begin{cases}x_{ \pm \alpha}^{\left(\frac{n}{p}\right)} \bullet{ }_{\phi} m & \text { si } p \mid n, \\
0 & \text { sinon }\end{cases} \\
& = \begin{cases}x_{ \pm \alpha}^{(n)} m & \text { si } p \mid n, \text { considérant cette fois } m \text { comme un élément de } M, \\
0 & \text { sinon, }\end{cases}
\end{aligned}
$$

et donc (3) devient $x_{ \pm \alpha}^{(n)} f_{m}=f_{x_{ \pm \alpha}^{(n)} \cdot m}$, et l'égalité (2) est donc vraie, comme souhaité.

2.3. Le foncteur de torsion par Frobenius des $G$-modules étant un adjoint à droite du foncteur $\left(?^{G_{1}}\right)^{[-1]}$, on a donc

Corollaire. (i) Le foncteur de contraction par Frobenius est un adjoint $\grave{a}$ droite du foncteur $\mathrm{St} \otimes \mathrm{St} \otimes\left(?^{[1]}\right)$ sur la catégorie des $G$-modules.

(ii) Si $M$ est un $G$-module injectif, $M^{\phi}$ l'est également.

Preuve. (i) Pour deux $G$-modules $M$ et $V$, on a

$$
\begin{aligned}
& G \operatorname{Mod}\left(\operatorname{St} \otimes \operatorname{St} \otimes V^{[1]}, M\right) \\
& \quad \simeq G \operatorname{Mod}\left(V^{[1]}, \operatorname{St} \otimes \operatorname{St} \otimes M\right) \\
& \quad \simeq G \operatorname{Mod}\left(V^{[1]},(\operatorname{St} \otimes \operatorname{St} \otimes M)^{G_{1}}\right) \\
& \quad \simeq G \operatorname{Mod}\left(V^{[1]},\left(\left((\operatorname{St} \otimes \operatorname{St} \otimes M)^{G_{1}}\right)^{\phi}\right)^{[1]}\right) \\
& \left.\simeq G \operatorname{Mod}\left(V,\left((\operatorname{St} \otimes \operatorname{St} \otimes M)^{G_{1}}\right)^{\phi}\right)\right) \\
& \quad \text { car le foncteur torsion par Frobenius est pleinement fidèle } \\
& \quad \simeq G \operatorname{Mod}\left(V, M^{\phi}\right) .
\end{aligned}
$$

(ii) C'est immédiat à partir de (i); en effet, $\mathrm{St} \otimes \mathrm{St} \otimes\left({ }^{[1]}\right)$ est un foncteur exact. On peut aussi, de manière alternative, remarquer que tout $G$-module injectif peut s'écrire comme somme directe de copies de $\mathbb{k}[G][\mathrm{J}, \mathrm{I} .3 .9]$ et il suffit juste alors de vérifier que $\left(\mathbb{k}[G]^{G_{1}}\right)^{[-1]}$ demeure injectif. On remarque pour ce faire qu'on a

$$
\begin{aligned}
\left(\mathbb{k}[G]^{G_{1}}\right)^{[-1]} & \simeq \mathbb{k}\left[G / G_{1}\right]^{[-1]} \quad[\mathrm{J}, \text { I.5.5.6] } \\
& \simeq\left(\mathbb{k}[G]^{[1]}\right)^{[-1]} \simeq \mathbb{k}[G]
\end{aligned}
$$

en utilisant le diagramme du (1.2)(iv)

2.4. Soit $r \in \mathbb{N}^{+}, \mathrm{St}_{r}=L\left(\left(p^{r}-1\right) \rho\right)$ le $r$-ième module de Steinberg. Itérant le foncteur de contraction par Frobenius, on obtient

Théorème. La r-ième contraction par Frobenius (?) $)^{\phi^{r}}$ est adjointe à droite du foncteur $\mathrm{St}_{r} \otimes \mathrm{St}_{r} \otimes\left(?^{[r]}\right)$. 


\section{$3^{\circ}$. Bonnes filtrations}

Pour tout $\lambda \in \Lambda^{+}$, rappelons qu'on dispose des modules standards ou modules de Weyl duaux $\nabla(\lambda)=\left\{g \in \mathbb{k}[G] \mid f(g b)=\lambda(b)^{-1} f(g), \forall g \in G, \forall b \in B\right\}$ ainsi que des modules de Weyl $\Delta(\lambda)$ de plus haut poids $\lambda \in \Lambda^{+}$[J, II.2.13 Rmk 1) II.8.8, 8.9]. On a, par exemple, $\mathrm{St}=\nabla((p-1) \rho)$. On peut développer la théorie de ces modules $\operatorname{sur} \mathbb{Z}$, i.e. partir du modèle, essentiellement unique, de Chevalley déployé $G_{\mathbb{Z}}$ de $G$ sur $\mathbb{Z}$, et définir des $\mathbb{Z}$-modules $\nabla_{\mathbb{Z}}(\lambda)$ et $\Delta_{\mathbb{Z}}(\lambda)$ redonnant les $\Delta(\lambda)$ et $\nabla(\lambda)$ par extension des scalaires. Les $\nabla_{\mathbb{Z}}(\lambda) \otimes_{\mathbb{Z}} \mathbb{Q}$ fournissent alors (avec les notations évidentes) les modules simples pour $G_{\mathbb{Z}} \otimes_{\mathbb{Z}} \mathbb{Q}$.

Nous dirons qu'un $G$-module de dimension finie $M$ admet une filtration de Weyl si et seulement si tous les sous-quotients correspondants de la filtration sont des modules de Weyl. Dualement, on dira que $M$ admet une bonne filtration si et seulement si tous les sous-quotients correspondants sont de la forme $\nabla(\lambda)$.

3.1. On va s'intéresser à l'existence de bonnes filtrations sur $M^{\phi}$. Rappelons qu'il existe [vdK, §3 ] un $G$-module $M$ admettant une bonne filtration tel que $\left(M^{G_{1}}\right)^{[-1}$ ] n'en admette pas. Néanmmoins, pour tout $G$-module, on a

$$
M^{\phi} \geq\left(M^{G_{1}}\right)^{\phi}=\left\{\left\{\left(M^{G_{1}}\right)^{[-1]}\right\}^{[1]}\right\}^{\phi}=\left(M^{G_{1}}\right)^{[-1]} .
$$

Rappelons aussi que l'application naturelle $\nabla(p \lambda)^{\phi} \rightarrow \nabla(\lambda)\left(\lambda \in \Lambda^{+}\right)$est naturellement scindée par l'application d'élévation à la puissance $p$-ième [GK11, Prop. 3.2], faisant donc de $\nabla(\lambda)$ un facteur direct de $\nabla(p \lambda)^{\phi}$.

Pour énoncer plus simplement les résultats qui vont suivre, nous adopterons par commodité la convention que le module nul admet à la fois une filtration de Weyl et une bonne filtration (l'ensemble de ces filtrations étant toutefois réduit à l'ensemble vide). Le résultat suivant améliore inconditionellement [GK15, Cor. 5.8.1].

Théorème. Soit Mun G-module de dimension finie. Si $M$ admet une bonne filtration, il en est de même de $M^{\phi}$.

Preuve. Il suffit de montrer que $\operatorname{Ext}^{1}\left(\Delta(\lambda), M^{\phi}\right)=0$ pour tout $\lambda \in \Lambda^{+}$ [J, II.4.16]. Mais

$$
\begin{aligned}
& \operatorname{Ext}^{1}\left(\Delta(\lambda), M^{\phi}\right) \\
& \simeq \operatorname{Ext}^{1}\left(\operatorname{St} \otimes \operatorname{St} \otimes \Delta(\lambda)^{[1]}, M\right) \\
& \quad \quad \text { car le foncteur de contraction par Frobenius est exact, } \\
& \simeq \operatorname{Ext}^{1}(\operatorname{St} \otimes \Delta((p-1) \rho+p \lambda), M) \\
& \quad \text { par la version duale d'un théorème de Andersen-Haboush [J, II.3.19] } \\
& =0 \quad \text { car St } \otimes \Delta((p-1) \rho+p \lambda) \text { admet une filtration de Weyl [J, II.4.21]. }
\end{aligned}
$$


3.2. Remarque. Utilisant la $G_{1}$-projectivité du module de Steinberg [J, II.10.2], Donkin réduit le problème à montrer que $G_{1} \operatorname{Mod}(\operatorname{St}, \nabla(\lambda))^{[-1]}$ reste un $G$-module standard s'il ne s'annule pas. S'il est non nul, le $G_{1}$-socle de $\nabla(\lambda)$ doit être dans le même bloc que celui de la représentation de Steinberg, et donc $\lambda=(p-1) \rho+p \lambda^{1}$ pour un certain $\lambda^{1} \in \Lambda^{+}$. On a alors $\nabla(\lambda) \simeq \operatorname{St} \otimes \nabla\left(\lambda^{1}\right)^{[1]}$ de nouveau grâce au théorème d'Andersen-Haboush [J, II.3.19], et $G_{1} \operatorname{Mod}(\operatorname{St}, \nabla(\lambda))^{[-1]} \simeq \nabla\left(\lambda^{1}\right)$ par $G_{1}$-simplicité de St.

3.3. Pour ce qui suit, nous noterons $W$ le groupe de Weyl attaché aux données du $1^{\circ}$ et $\bullet$ son action sur $\Lambda$ définie par $w \bullet \mu=w(\mu+\rho)-\rho$ pour tous $w \in W, \mu \in \Lambda$. Pour tout $G$-module de dimension finie $M$ admettant une bonne filtration et $\lambda \in \Lambda^{+}$, nous noterons $(M: \nabla(\lambda))$ la multiplicité de $\nabla(\lambda)$ dans celle-ci. Nous noterons $\mathbb{B}(\lambda)$ l'ensemble des chemins de Lakshmibai-Seshadri de forme $\lambda$ [Li94, 2, Def]. Ils nous serviront juste à montrer comment éviter la somme alternée dans l'énoncé suivant.

Corollaire. (i) Pour tout $G$-module de dimension finie $M$ admettant une bonne filtration et tous $\lambda, \mu \in \Lambda^{+}$, on a

$$
\begin{aligned}
\left(\nabla(\lambda)^{\phi}: \nabla(\mu)\right) & =(\operatorname{St} \otimes \nabla((p-1) \rho+p \mu): \nabla(\lambda))=(\operatorname{St} \otimes \nabla(\lambda): \nabla((p-1) \rho+p \mu)) \\
& =\sum_{w \in W}(-1)^{\ell(w)} \operatorname{dim} \nabla(\lambda)_{p(w \bullet \mu)} \\
& =\#\{\pi \in \mathbb{B}(\lambda) \mid \pi \text { est }(p-1) \rho \text {-dominant et } \pi(1)=p \mu\}
\end{aligned}
$$

En particulier, si $\mu \in \Lambda^{+}$est maximal parmi ceux des $\nu \in \Lambda$ tels que p $\nu$ soit un poids de $\nabla(\lambda)$, on $a$

$$
(\mathrm{St} \otimes \nabla(\lambda): \nabla((p-1) \rho+p \mu))=\operatorname{dim} \nabla(\lambda)_{p \mu} .
$$

(ii) Un G-module de basculement ("tilting module" [J, E.1]) admet une contraction par Frobenius qui est G-module de basculement.

Preuve. (i) On a

$$
\begin{aligned}
\left(\nabla(\lambda)^{\phi}\right. & : \nabla(\mu))=\operatorname{dim} G \operatorname{Mod}\left(\Delta(\mu), \nabla(\lambda)^{\phi}\right) \quad \text { grâce à }[J, \text { II.4.16] } \\
& =\operatorname{dim} G \operatorname{Mod}\left(\operatorname{St} \otimes \operatorname{St} \otimes \Delta(\mu)^{[1]}, \nabla(\lambda)\right) \\
& =\operatorname{dim} G \operatorname{Mod}(\operatorname{St} \otimes \Delta((p-1) \rho+p \mu), \nabla(\lambda))=(\operatorname{St} \otimes \nabla((p-1) \rho+p \mu): \nabla(\lambda)) \\
& =\operatorname{dim} G \operatorname{Mod}(\Delta((p-1) \rho+p \mu), \operatorname{St} \otimes \nabla(\lambda))=(\operatorname{St} \otimes \nabla(\lambda): \nabla((p-1) \rho+p \mu)) .
\end{aligned}
$$

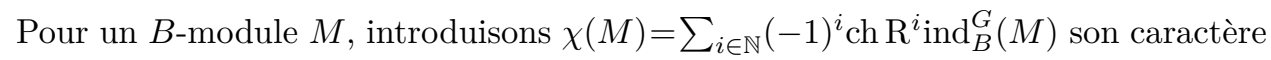
d'Euler [J, II.5.7], ch désignant le caractère formel [J, I.2.11(6)]. Alors 


$$
\begin{aligned}
\operatorname{ch} & (\operatorname{St} \otimes \nabla(\lambda)) \\
= & \operatorname{ch} \nabla((p-1) \rho \otimes \nabla(\lambda)) \quad \text { grâce à l'identité tensorielle [J, I.3.6] } \\
=\chi((p-1) \rho \otimes \nabla(\lambda)) & \begin{array}{l}
\text { grâce au théorème d'annulation de Kempf ou même } \\
\text { simplement par le principe de strong linkage [J, II.6.15] }
\end{array} \\
& =\sum_{\nu \in \Lambda} \chi((p-1) \rho+\nu) \operatorname{dim} \nabla(\lambda)_{\nu} \quad \text { car } \chi \text { est additive. }
\end{aligned}
$$

Pour $w \in W$ on a $\chi\left(w^{-1} \bullet((p-1) \rho+\nu)\right)=(-1)^{\ell(w)} \chi((p-1) \rho+\nu)$, et $w^{-1} \bullet((p-1) \rho+$ $\nu)=(p-1) \rho+p \mu$ si et seulement si $\nu=p(w \bullet \mu)$. Les composants de $\operatorname{ch}(\operatorname{St} \otimes \nabla(\lambda))$ contribuant à la multiplicité de $(\operatorname{St} \otimes \nabla(\lambda): \nabla((p-1) \rho+p \mu))$ sont donc juste les

$$
\sum_{w \in W} \chi(w \bullet((p-1) \rho+p \mu)) \operatorname{dim} \nabla(\lambda)_{p(w \bullet \mu)},
$$

et par suite $(\operatorname{St} \otimes \nabla(\lambda): \nabla((p-1) \rho+p \mu))=\sum_{w \in W}(-1)^{\ell(w)} \operatorname{dim} \nabla(\lambda)_{p(w \bullet \mu)}$.

(ii) découle du théorème et de $(1.2 . \mathrm{v})$.

3.4. Montrons maintenant la nécessité des hypothèses de caractéristique puis de petitesse du plus haut poids dans la proposition 1.3 sur l'exemple d'un groupe $G$ de type $\mathrm{G}_{2}$ dont nous noterons $\alpha$ et $\beta$ les racines simples (avec $\alpha$ la courte) et $\varpi_{\alpha}$ et $\varpi_{\beta}$ les poids fondamentaux correspondants.

(i) Supposons que $p=2$. Les poids de $\mathrm{St}=L(\rho)$ appartenant à $2 \Lambda^{+}$sont juste $2 \varpi_{\alpha}$ et 0 de multiplicités respectivement 2 et 4 . Par $(3.1), \mathrm{St}^{\phi}=\nabla(\rho)^{\phi}$ admet une bonne filtration avec comme sous-quotients $\nabla\left(\varpi_{\alpha}\right)$ et $\mathbb{k}$ apparaissant chacun deux fois. Cependant $\nabla\left(\varpi_{\alpha}\right)$ n'est pas simple, ayant $\mathbb{k}$ comme module de tête par la formule des sommes de Jantzen [J, II.8.19]. Il s'ensuit que $L(\rho)^{\phi}$ ne peut être semi-simple dans ce cas.

(ii) Revenons à la situation et aux notations générales de (1.3). Même si $L\left(\lambda^{0}\right)^{\phi}$ reste semi-simple, $\left\{L\left(\lambda^{0}\right) \otimes L\left(\lambda^{1}\right)^{[1]}\right\}^{\phi} \simeq L\left(\lambda^{0}\right)^{\phi} \otimes L\left(\lambda^{1}\right)$ n'a aucune raison de l'être. En effet, considérons $L(\lambda) \otimes \mathrm{St}^{[1]}$ avec $\lambda=(p-3) \varpi_{\alpha}+2 \varpi_{\beta}$ en caractéristique $p \geq 3$. Comme $p \varpi_{\alpha}=\lambda-\beta$ est un poids de $\nabla(\lambda)$, c'en est également un de $L(\lambda)$; si $L(\mu)$ désigne un autre facteur de composition de $\nabla(\lambda)$, alors $\lambda-\beta \not \leq \mu$. Ainsi, $\varpi_{\alpha}$ est un poids maximal de $L(\lambda)^{\phi}$, et par suite $L(\lambda)^{\phi}$ a un facteur de composition $L\left(\varpi_{\alpha}\right)$. Comme $L\left(\varpi_{\alpha}\right)=\nabla\left(\varpi_{\alpha}\right)$ pour $p \neq 2,\left(L(\lambda) \otimes \mathrm{St}^{[1]}\right)^{\phi} \simeq L(\lambda)^{\phi} \otimes$ St admet un quotient $\nabla\left(p \varpi_{\alpha}+(p-1) \varpi_{\beta}\right)$. Mais $\nabla\left(p \varpi_{\alpha}+(p-1) \varpi_{\beta}\right)$ a un facteur de composition $L\left(p \varpi_{\alpha}+\right.$ $\left.(p-1) \varpi_{\beta}-\alpha\right)$, et n'est donc pas semi-simple.

\section{$4^{\circ} \cdot G_{r}$-modules}

Soit $r \in \mathbb{N}$ avec $r \geq 2$. 
4.1. La contraction par Frobenius induit un foncteur de la catégorie des $G_{r}$-modules dans celle des $G_{r-1}$-modules, que l'on continue de noter ${ }^{\phi}$.

Théorème. Il existe un isomorphisme de foncteurs $?^{\phi} \rightarrow\left\{(\mathrm{St} \otimes \mathrm{St} \otimes ?)^{G_{1}}\right\}^{[-1]}$ de la catégorie des $G_{r}$-modules dans celle des $G_{r-1}$-modules.

Preuve. Soit $M$ un $G_{r}$-module. On montre que $M^{\phi} \rightarrow G_{1} \operatorname{Mod}(\operatorname{St} \otimes \operatorname{St}, M)^{[-1]}$ via $m \mapsto f_{m}$ est $G_{r-1}$-linéaire. Comme $G_{r-1}$ n'est pas, en général, engendré par les $\left.x_{ \pm \alpha}^{(n)}, \alpha \in R^{\mathrm{s}}, n \in\right] 0, p^{r-1}[$, l'argument pour les $G$-modules ne se décalque pas tel quel. Néanmmoins, on peut supposer $M$ de dimension finie. Alors, comme les deux foncteurs de l'énoncé sont exacts, on a seulement à vérifier la $G_{r-1}$-linéarité de l'application ci-dessus sur les $G_{r}$ - modules simples. Mais ces derniers sont équipés d'une structure de $G$-modules, et l'on sait déjà que l'application est $G$-linéaire par (2.1) et donc, a fortiori, $G_{r-1}$-linéaire.

4.2. De même pour les $G_{r} T$-modules et pour les $G_{r} B$-modules.

Corollaire. Il existe un isomorphisme de foncteurs ${ }^{\phi} \rightarrow\left\{(\mathrm{St} \otimes \mathrm{St} \otimes ?)^{G_{1}}\right\}^{[-1]}$ de la catégorie des $G_{r} T$-(resp. $\left.G_{r} B-\right)$ modules dans celle des $G_{r-1} T$-(resp. $\left.G_{r} B-\right)$ modules.

Preuve. Soit $M$ un $G_{r} T$-module. L'application $m \mapsto f_{m}$ de est $T$-linéaire; si $m \in M_{p \lambda}$, on a $t m=\lambda(t) m$. Regardant $f_{m}$ comme un élément de $G_{1} \operatorname{Mod}(\operatorname{St} \otimes \operatorname{St}, M)$, $t f_{m}=t f_{m}\left(t^{-1}\right.$ ?). Comme $t f_{m}\left(t^{-1}\left(v_{+} \otimes v_{-}\right)\right)=t m=(p \lambda)(t) m, t f_{m}=(p \lambda)(t) f_{m}$, et donc $f_{m}$ a comme poids $\lambda$ comme élément de $G_{1} \operatorname{Mod}(\operatorname{St} \otimes \operatorname{St}, M)^{[-1]}$. Comme l'application ci-dessus est $G_{r-1}$-linéaire grâce au théorème, l'assertion pour les $G_{r} T$-modules s'ensuit. En ce qui concerne les $G_{r} B$-modules, $\operatorname{Dist}(U)$ est engendré par $x_{-\alpha}^{(n)}, \alpha \in R^{\mathrm{s}}, n \in \mathbb{N}$, et donc l'application est $\operatorname{Dist}(U)$-linéaire par (2.1). L'assertion s'ensuit.

4.3. Il découle de tout cela que la contraction par Frobenius sur les categories des $G_{r}$-modules, $G_{r} T$-modules, $G_{r} B$-modules sont adjoints à droite de la torsion par Frobenius tensorisé deux fois avec le module de Steinberg. Plus généralement:

Corollaire. Soit $s \in \mathbb{N}$ avec $0<s<r$.

(i) Le foncteur de s-ième contraction par Frobenius ? $^{s}$ de la catégorie des $G_{r^{-}}$ (resp. $\left.G_{r} T-, G_{r} B-\right)$ modules dans celle des $G_{r-s}-\left(r e s p . G_{r-s} T-, G_{r-s} B-\right)$ modules est adjoint à droite du foncteur $\mathrm{St}_{s} \otimes \mathrm{St}_{s} \otimes\left(?^{[s]}\right)$ de la catégorie des $G_{r-s}$-(resp. $\left.G_{r-s} T-, G_{r-s} B-\right)$ modules dans celle des $G_{r}$-(resp. $\left.G_{r} T-, G_{r} B-\right)$ modules.

(ii) Les foncteurs $?^{\phi^{s}}$ sur ces catégories préservent l'injectivité et la projectivité des modules de dimension finie. 
4.4. Pour un $B_{r^{-}}\left(\right.$resp. $\left.B_{r} T-, B_{-}\right)$module $M$ posons $\nabla_{r}(M)=\operatorname{ind}_{B_{r}}^{G_{r}}(M)$ (resp. $\left.\widehat{\nabla}_{r}(M)=\operatorname{ind}_{B_{r} T}^{G_{r} T}(M), \widetilde{\nabla}_{r}(M)=\operatorname{ind}_{B}^{G_{r} B}(M)\right)$. On dira qu'un $G_{r^{-}}\left(\operatorname{resp} . G_{r} T-, G_{r} B-\right)$ module $V$ de dimension finie admet une $\nabla_{r^{-}}\left(\right.$resp. $\left.\widehat{\nabla}_{r^{-}}, \widetilde{\nabla}_{r^{-}}\right)$filtration si et seulement si $V$ admet une $G_{r}$ (resp. $G_{r} T-, G_{r} B$-) filtration dont tous les sous-quotients correspondants sont de la forme $\nabla_{r}(\lambda)$ (resp. $\left.\widehat{\nabla}_{r}(\lambda), \widetilde{\nabla}_{r}(\lambda)\right), \lambda \in \Lambda$. Pour un $B_{r}^{+} T$-module $M^{\prime}$, posons aussi $\hat{\Delta}_{r}\left(M^{\prime}\right)=\operatorname{coind}_{B_{r}^{+} T}^{G_{r}^{+} T}\left(M^{\prime}\right)$ [J, II.9.1.5].

Rappelons [J, II.11.2] qu'un $G_{r} T$-module $M$ de dimension finie admet une $\widehat{\nabla}_{r}$-filtration si et seulement si $M$ est $B_{r}^{+} T$-injectif. Comme pour les $G$-modules, on a

Lemme. Un $G_{r} T$-module $M$ de dimension finie admet une $\widehat{\nabla}_{r}$-filtration si et seulement si $\operatorname{Ext}_{G_{r} T}^{1}\left(\hat{\Delta}_{r}(\lambda), M\right)=0$ pour tout $\lambda \in \Lambda$.

Preuve. Supposons tout d'abord que $\operatorname{Ext}_{G_{1} T}^{1}(\hat{\Delta}(\lambda), M)=0$ pour tout $\lambda \in \Lambda$. Si $Q$ est une $B_{1}^{+} T$-enveloppe injective du $B_{1}^{+} T$-socle de $M$, on veut voir que le monomorphisme $M \hookrightarrow Q$ est scindé, ce qui découlera de $\operatorname{Ext}_{B_{1}^{+} T}^{1}(Q / M, M)=0$. Pour avoir cela, il suffit de vérifier que $\operatorname{Ext}_{B_{1}^{+} T}^{1}(\lambda, M)=0$ pour tout $\lambda \in \Lambda$. Mais

$$
\begin{aligned}
\operatorname{Ext}_{B_{1}^{+} T}^{1}(\lambda, M) & \simeq \operatorname{Ext}_{B_{1}^{+} T}^{1}\left(M^{*},-\lambda\right) \simeq \operatorname{Ext}_{G_{1} T}^{1}\left(M^{*}, \operatorname{ind}_{B_{1}^{+} T}^{G_{1} T}(-\lambda)\right) \\
& \simeq \operatorname{Ext}_{G_{1} T}^{1}\left(M^{*}, \hat{\Delta}(-\lambda+2(p-1) \rho)\right) \quad \text { grâce à }[J, \text { II.9.2] } \\
& \simeq \operatorname{Ext}_{G_{1} T}^{1}\left(\hat{\Delta}(-\lambda+2(p-1) \rho)^{*}, M\right) \\
& \simeq \operatorname{Ext}_{G_{1} T}^{1}(\hat{\Delta}(\lambda), M) \quad \text { de nouveau grâce à [J, II.9.2] } \\
& =0 .
\end{aligned}
$$

La réciproque vaut également.

4.5. Soit $s \in \mathbb{N}$ avec $0<s<r$.

Théorème. Si $M$ est un $G_{r} T$-module de dimension finie admettant une $\widehat{\nabla}_{r}$-filtration, $M^{\phi^{s}}$ admet une $\widehat{\nabla}_{r-s}$-filtration.

Preuve. Par (4.4) il suffit simplement de voir que $\operatorname{Ext}_{G_{r-s} T}^{1}\left(\hat{\Delta}_{r-s}(\lambda), M^{\phi^{s}}\right)=0$ pour tout $\lambda \in \Lambda$. Par (4.3) $\operatorname{Ext}_{G_{r-s} T}^{1}\left(\hat{\Delta}_{r-s}(\lambda), M^{\phi^{s}}\right) \simeq \operatorname{Ext}_{G_{r} T}^{1}\left(\operatorname{St}_{s} \otimes \operatorname{St}_{s} \otimes \hat{\Delta}_{r-s}(\lambda)^{[s]}\right.$, $M)$. Maintenant $\hat{\Delta}_{r-s}(\lambda)$ s'étend en $\tilde{\Delta}_{r-s}(\lambda)$ [J, II.9.1.4], et, comme $G$-modules,

$$
\begin{aligned}
\mathrm{St}_{s} & \otimes \tilde{\Delta}_{r-s}(\lambda) \\
& \simeq \mathrm{St}_{s} \otimes \operatorname{ind}_{B^{+}}^{G_{r-s} B^{+}}\left(\lambda-2\left(p^{r-s}-1\right) \rho\right)^{[s]} \quad \text { grâce à }[\mathrm{J}, \mathrm{II} .9 .2] \\
& \simeq \mathrm{St}_{s} \otimes \operatorname{ind}_{G_{s} B^{+} / G_{s}}^{G_{r} B^{+} / G_{s}}\left(p^{s}\left(\lambda-2\left(p^{r-s}-1\right) \rho\right)\right)
\end{aligned}
$$




$$
\begin{array}{ll}
\simeq \operatorname{St}_{s} \otimes \operatorname{ind}_{G_{s} B^{+}}^{G_{r} B^{+}}\left(p^{s}\left(\lambda-2\left(p^{r-s}-1\right) \rho\right)\right) & \text { grâce à [J, II.3.19] } \\
\simeq \operatorname{ind}_{G_{s} B^{+}}^{G_{r} B^{+}}\left(\operatorname{St}_{s} \otimes p^{s}\left(\lambda-2\left(p^{r-s}-1\right) \rho\right)\right) & \text { grâce à l'identité tensorielle [J, I.3.6] } \\
\simeq \operatorname{ind}_{G_{s} B^{+}}^{G_{r} B^{+}}\left(\operatorname{ind}_{B^{+}}^{G_{s} B^{+}}\left(\left(p^{s}-1\right) \rho-2\left(p^{s}-1\right) \rho\right) \otimes p^{s}\left(\lambda-2\left(p^{r-s}-1\right) \rho\right)\right) & \text { de nouveau grâce à [J, II.9.2] } \\
\simeq \operatorname{ind}_{G_{s} B^{+} B_{r} B^{+}}\left(\operatorname{ind}_{B^{+}}^{G_{s} B^{+}}\left(-\left(p^{s}-1\right) \rho+p^{s}\left(\lambda-2\left(p^{r-s}-1\right) \rho\right)\right)\right) \\
\simeq \operatorname{ind}_{B^{+}}^{G_{r} B^{+}}\left(\left(p^{s}-1\right) \rho+p^{s} \lambda-2\left(p^{r}-1\right) \rho\right) \\
\simeq \hat{\Delta}_{r}\left(\left(p^{s}-1\right) \rho+p^{s} \lambda\right) \quad \text { grâce à }[\mathrm{J}, \mathrm{II} .9 .2] .
\end{array}
$$

Ainsi

$$
\begin{aligned}
\mathrm{St}_{s} \otimes \mathrm{St}_{s} \otimes \hat{\Delta}_{r-s}(\lambda)^{[s]} & \simeq \mathrm{St}_{s} \otimes \hat{\Delta}_{r}\left(\left(p^{s}-1\right) \rho+p^{s} \lambda\right) \\
& \simeq \hat{\Delta}_{r}\left(\mathrm{St}_{s} \otimes\left(\left(p^{s}-1\right) \rho+p^{s} \lambda\right)\right)
\end{aligned}
$$

grâce à l'identité tensorielle [J, I.3.6]

admet une $\hat{\Delta}_{r}$-filtration, et donc $\operatorname{Ext}_{G_{r} T}^{1}\left(\operatorname{St}_{s} \otimes \operatorname{St}_{s} \otimes \hat{\Delta}_{r-s}(\lambda)^{[s]}, M\right)=0$ par (4.4), comme voulu.

\subsection{Soit $s \in \mathbb{N}$ avec $0<s<r$.}

Corollaire. Si $M$ est un $G_{r}$-(resp. $\left.G_{r} B-\right)$ module de dimension finie admettant une $\nabla_{r^{-}}\left(\right.$resp. $\left.\widetilde{\nabla}_{r^{-}}\right)$filtration, $M^{\phi^{s}}$ admet une $\nabla_{r-s}-\left(\right.$ resp. $\left.\widetilde{\nabla}_{r-s^{-}}\right)$filtration.

Preuve. Pour les $G_{r}$-modules, il suffit de voir que chaque $\nabla_{r}(\lambda)^{\phi^{s}}, \lambda \in \Lambda$, admet une $\nabla_{r-s}$-filtration. Mais $\nabla_{r}(\lambda)$ s'étend à $\widehat{\nabla}_{r}(\lambda)$, et l'assertion découle donc de (4.5). Pour les $G_{r} B$-modules il suffit de nouveau de voir que chaque $\widetilde{\nabla}_{r}(\lambda)^{\phi^{s}}$, $\lambda \in \Lambda$, admet une $\widetilde{\nabla}_{r-s}$-filtration. Comme $\widetilde{\nabla}_{r}(\lambda)=\widehat{\nabla}_{r}(\lambda)$ en tant que $G_{r} T$-modules, $\widetilde{\nabla}_{r}(\lambda)^{\phi^{s}}$ admet une $\widehat{\nabla}_{r-s}$-filtration par $(4.5)$, et donc $\widetilde{\nabla}_{r}(\lambda)=\widehat{\nabla}_{r}(\lambda)$ est $B_{r-s}^{+} T$ injectif par (4.4). Le module $\widetilde{\nabla}_{r}(\lambda)^{\phi^{s}}$ admet donc une $\widetilde{\nabla}_{r-s}$-filtration par [J, Rmk. II.11.2.2].

\section{References}

[D] Donkin, S., Hopf complements and injective comodules for algebraic groups, Proc. London Math. Soc. 40 (1980), 298-319.

[GK11] Gros, M. and Kaneda, M., Contraction par Frobenius de G-modules, Ann. Inst. Fourier (Grenoble) 61 (2011), 2507-2542. 
[GK] Gros, M. and Kaneda, M., Un scindage du morphisme de Frobenius quantique. arXiv: 1302.2437.

[GK15] Gros, M. and KanedA, M., Un scindage du morphisme de Frobenius quantique, Ark. Mat. 53 (2015), 271-301.

[J73] Jantzen, J. C., Darstellungenhalbeinfacher algebraischer Gruppen und zugeordnet kontravariante Formen, Bonner Math. Schriften 67 (1973).

[J] Jantzen, J. C., Representations of Algebraic Groups, 2003, American Math. Soc.

[Li94] Littelmann, P., A Littlewood-Richardson rule for symmetrizable Kac-Moody algebras, Invent. Math. 116 (1994), 329-346.

[Li98] Littelmann, P., Contracting modules and standard monomial theory for symmetrizable Kac-Moody algebras, J. Amer. Math. Soc. 11 (1998), 551-567.

[NT] Nagao, H. and Tsushima, Y., Representations of Finite Groups, Acad. Press, 1989.

[vdK] van der KALLEN, W., Infinitesimal fixed points in modules with good filtration, Math. Z. 212 (1993), 157-159.

Michel Gros

CNRS UMR 6625

IRMAR

Université de Rennes 1

Campus de Beaulieu

FR-35042

Rennes cedex

France

michel.gros@univ-rennes1.fr
Kaneda Masaharu

Osaka City University

Department of Mathematics

JP-558-8585

Osaka

Japan

kaneda@sci.osaka-cu.ac.jp

Received March 8, 2017

in revised form August 7, 2017

Ajouté sur épreuves: Le cas d'un groupe réductif général pour lequel le module de Steinberg n'est pas disponible est traité dans notre article "Contraction par Frobenius pour les groupes réductifs". 